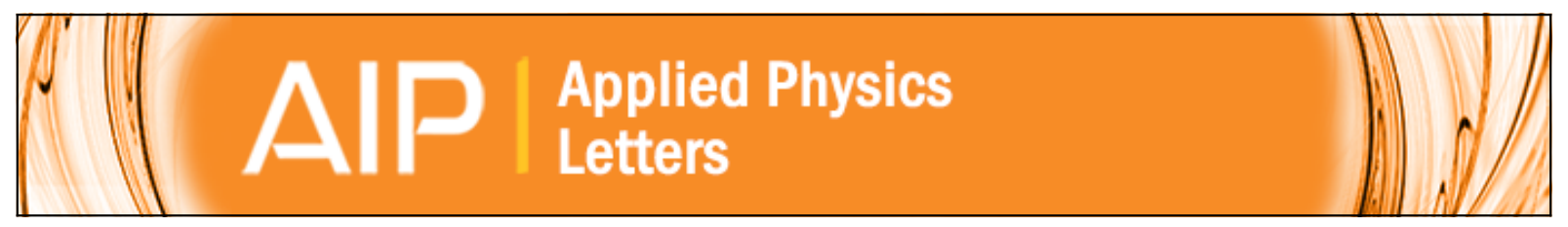

\title{
Atomic relocation processes in impurity-free disordered $p$-GaAs epilayers studied by deep level transient spectroscopy
}

P. N. K. Deenapanray, A. Martin, S. Doshi, H. H. Tan, and C. Jagadish

Citation: Applied Physics Letters 81, 3573 (2002); doi: 10.1063/1.1519728

View online: http://dx.doi.org/10.1063/1.1519728

View Table of Contents: http://scitation.aip.org/content/aip/journal/apl/81/19?ver=pdfcov

Published by the AIP Publishing

\section{Articles you may be interested in}

Deep level photothermal spectroscopy: Physical principles and applications to semi-insulating GaAs band-gap multiple trap states

J. Appl. Phys. 103, 043704 (2008); 10.1063/1.2842401

Effects of thermal annealing on deep-level defects and minority-carrier electron diffusion length in Be-doped InGaAsN

J. Appl. Phys. 97, 073702 (2005); 10.1063/1.1871334

Observed trapping of minority-carrier electrons in $\mathrm{p}$-type GaAsN during deep-level transient spectroscopy measurement

Appl. Phys. Lett. 86, 072109 (2005); 10.1063/1.1865328

Towards a better understanding of the operative mechanisms underlying impurity-free disordering of GaAs:

Effect of stress

J. Vac. Sci. Technol. B 21, 198 (2003); 10.1116/1.1535927

Deep levels in low temperature GaAs probed by field effect deep level transient spectroscopy

J. Vac. Sci. Technol. B 17, 60 (1999); 10.1116/1.590517

\section{AlP $\left.\right|_{\text {APL Photonics }}$}

APL Photonics is pleased to announce Benjamin Eggleton as its Editor-in-Chief

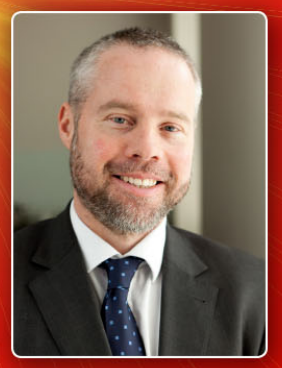




\title{
Atomic relocation processes in impurity-free disordered $p$-GaAs epilayers studied by deep level transient spectroscopy
}

\author{
P. N. K. Deenapanray, ${ }^{\text {a) }}$ A. Martin, ${ }^{\text {b) }}$ S. Doshi, H. H. Tan, and C. Jagadish \\ Department of Electronic Materials Engineering, Research School of Physical Sciences and Engineering, \\ The Australian National University, Canberra, ACT 0200, Australia
}

(Received 30 May 2002; accepted 16 September 2002)

\begin{abstract}
We have used capacitance-voltage and deep level transient spectroscopy techniques to study the relocation of impurities, such as $\mathrm{Zn}$ and $\mathrm{Cu}$, in impurity-free disordered (IFD) $p$-type GaAs. A four-fold increase in the doping concentration is observed after annealing at $925^{\circ} \mathrm{C}$. Two electrically active defects $\mathrm{HA}\left(E_{V}+0.39 \mathrm{eV}\right)$ and $\mathrm{HB} 2\left(E_{V}+0.54 \mathrm{eV}\right)$, which we have attributed to $\mathrm{Cu}$ - and $\mathrm{As}_{i} / \mathrm{As}_{\mathrm{Ga}}$-related levels, respectively, are observed in the disordered $p$-GaAs layers. The injection of gallium vacancies causes segregation of $\mathrm{Zn}$ dopant atoms and $\mathrm{Cu}$ towards the surface of IFD samples. The atomic relocation process is critically assessed in terms of the application of IFD to the band gap engineering of doped GaAs-based heterostructures. (c) 2002 American Institute of Physics. [DOI: 10.1063/1.1519728]
\end{abstract}

The selective area band gap modification of III-V semiconductor compounds is a key requirement for achieving monolithic integration of optoelectronic and photonic devices. Impurity-free disordering (IFD) using dielectric capping layers has been actively researched for the past two decades as a promising, technologically simpler alternative to regrowth and selective area epitaxy. However, the technological simplicity of IFD masks an underlying complex process that depends on several factors, including type and quality of capping layer (related to deposition technique and process parameters), ${ }^{1-4}$ stress, ${ }^{5,6}$ annealing ambient, ${ }^{7}$ and doping. ${ }^{8-10}$

In an attempt to understand the defect reactions that drive the disordering process, we have recently investigated the electronic and annealing properties of the defects created in $n$-type GaAs epilayers by IFD. ${ }^{11-13}$ Previous studies of IFD have concentrated on the use of undoped heterostructures, ${ }^{1-6}$ and the results have been automatically applied to practical devices containing doped epilayers. IFD of doped structures may result in unwanted migration of dopant atoms, such as $\mathrm{Zn}$ and $\mathrm{Be}$, which causes spatially nonselective impurity-induced disordering. ${ }^{7-10}$ There is the need, therefore, to investigate the relocation of impurities in IFDdoped structures. In this letter, we report on the segregation of impurities, including $\mathrm{Zn}$ dopant atoms and $\mathrm{Cu}$, in rapid thermally annealed $\mathrm{SiO}_{2}$-capped $p$ GaAs. We discuss the impurity relocation process and its implications regarding the technological viability of IFD.

Epitaxial GaAs (100) layers doped with $\sim 9$ $\times 10^{15} \mathrm{Zn} / \mathrm{cm}^{3}$ were grown by metalorganic chemical vapor deposition at $750{ }^{\circ} \mathrm{C}$. The $4 \mu \mathrm{m}$ thick epitaxial layers were capped with $200 \mathrm{~nm} \mathrm{SiO}_{2}$ by plasma-enhanced chemical vapor deposition. Rapid thermal annealing (RTA) was performed on $\mathrm{SiO}_{2}$-capped samples for $30 \mathrm{~s}$ in the $800^{\circ} \mathrm{C}-925^{\circ} \mathrm{C}$ range under Ar flow. Following removal of

\footnotetext{
${ }^{a)}$ Electronic mail: pnk109@ @sphysse.anu.edu.au

${ }^{b)}$ Also at: Laboratoire de Physique des Interfaces et Couches Minces, Ecole Polytechnique, 91128 Palaiseau Cedex, France.
}

the $\mathrm{SiO}_{2}$ layers, the GaAs layers were chemically cleaned prior to the fabrication of Ti Schottky barrier diodes, $200 \mathrm{~nm}$ thickness and $0.77 \mathrm{~mm}$ diameter, by electron-beam evaporation. Ohmic contacts were made to the back side of samples using an In-Ga eutectic. High-frequency (1 $\mathrm{MHz})$ capacitance-voltage $(C-V)$ and deep level transient spectroscopy (DLTS) measurements were used to characterize samples.

Figure 1 illustrates the doping concentration, $N_{\mathrm{A}}$, determined from $C-V$ measurements at room temperature, in the as-grown (open diamond) and impurity-free disordered $p$-GaAs epilayers. The disordered epilayers exhibit a higher free carrier concentration that increases with the increasing annealing temperature. A nearly four-fold increase in the free carrier concentration is observed following annealing at $925^{\circ} \mathrm{C}$. The increase in the doping concentration shown in Fig. 1 may arise from either (a) the segregation of $\mathrm{Zn}$ dopant atoms, and/or (b) an increase in acceptor-type defects in the near-surface region. The uncapped and annealed samples did not exhibit significant changes in doping concentration, and

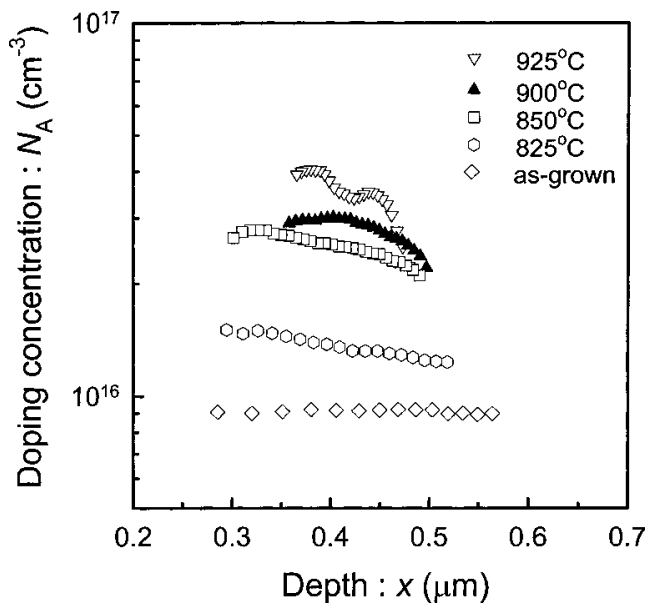

FIG. 1. Doping profiles of $p$-GaAs disordered at different annealing temperatures. The profiles are extracted from room temperature $C-V$ measurements. 


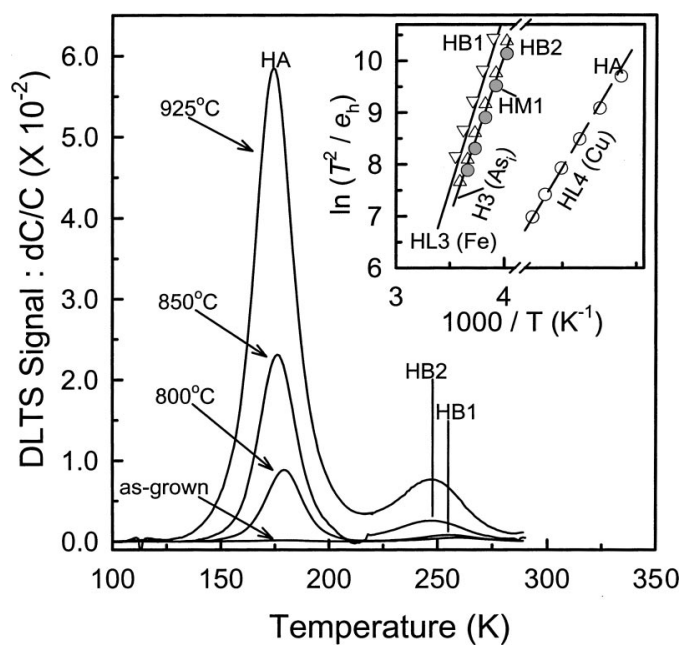

FIG. 2. DLTS spectra measured from as-grown and disordered $p$-GaAs samples. The spectrum taken from the as-grown sample is also shown. The inset shows the Arrhenius-type plots (open symbols) from which the signatures of defects are extracted. The solid line and solid circles overlaying the experimental data points are signatures of $\mathrm{Cu}-, \mathrm{Fe}-$, and EL2-related defects previously reported in $p$-GaAs.

we exclude purely thermal effects as the cause for the changes in $N_{\mathrm{A}}$.

We first assess the influence of annealing temperature on the creation of electrically active defects in the $p$-GaAs samples. The DLTS spectra shown in Fig. 2 were measured from both the as-grown and disordered $p$-GaAs for selected RTA temperatures. The region extending between $\sim 0.43$ to $\sim 0.57 \mu \mathrm{m}$ was probed by DLTS. The inset in Fig. 2 illustrates the Arrhenius plots of $\ln \left(T^{2} / e_{\mathrm{h}}\right)$ versus $1000 / T$ from which the activation energy, $E_{T}$, and apparent capture cross section, $\sigma_{a}$, of defects were extracted (i.e., "signature"). The open symbols correspond to our experimental data points, while the lines (HL4, HL3, and H3) and solid circles (HM1) are the signatures of defects previously reported in the literature. ${ }^{14-16}$ This comparison allows the tentative identification of our defects. The as-grown layers contained defects HA $\left(E_{T} \sim E_{V}+0.39 \mathrm{eV} ; \sigma_{a} \sim 6.3 \times 10^{-15} \mathrm{~cm}^{-2}\right)$ and HB1 $\left(E_{T} \sim E_{V}+0.57 \mathrm{eV} ; \sigma_{a} \sim 3.0 \times 10^{-15} \mathrm{~cm}^{-2}\right)$ in concentrations $\sim 2 \times 10^{12} \mathrm{~cm}^{-3}$ and $\sim 4 \times 10^{12} \mathrm{~cm}^{-3}$, respectively. HA and HB1 are normally observed in metalorganic chemical vapor deposition (MOCVD) grown $p$-GaAs epilayers and can be identified as $\mathrm{Cu}$ - (HL4) and Fe-related (HL3) defects, respectively. ${ }^{14}$ It is pointed out here that although the single donor state $(0 /+)$ of $\mathrm{As}_{\mathrm{Ga}}$ (EL2) is always detected in MOCVD $n$-GaAs, ${ }^{11}$ the double donor state of EL2 $(+/$ $++)\left(E_{V}+0.54 \mathrm{eV}\right)^{16}$ has not been measured in $p$-GaAs epitaxial layers to date. In Ref. 16, a defect HM1 was shown to be the double donor state of the EL2, and the inset in Fig. 2 shows that $\mathrm{HB} 1$ is not similar to HM1.

Figure 2 shows that a defect HB2 $\left(E_{T} \sim E_{V}+0.55 \mathrm{eV}\right.$; $\sigma_{a} \sim 2.7 \times 10^{-15} \mathrm{~cm}^{-2}$ ) with slightly lower activation energy than $\mathrm{HB} 1$ is created by $\mathrm{SiO}_{2}$-capped annealing. The peak position of HB2 is shifted to the lower temperature by $\sim 6 \mathrm{~K}$ compared to HB1. Further, the peak intensity of HA and HB2 increase with the increasing annealing temperature. The signature of HB2 is identical to defect $\mathrm{H} 3$ in electron irradiated $p$-GaAs epilayers. ${ }^{15}$ In that study, meticulous fluence dependence and annealing experiments convincingly showed

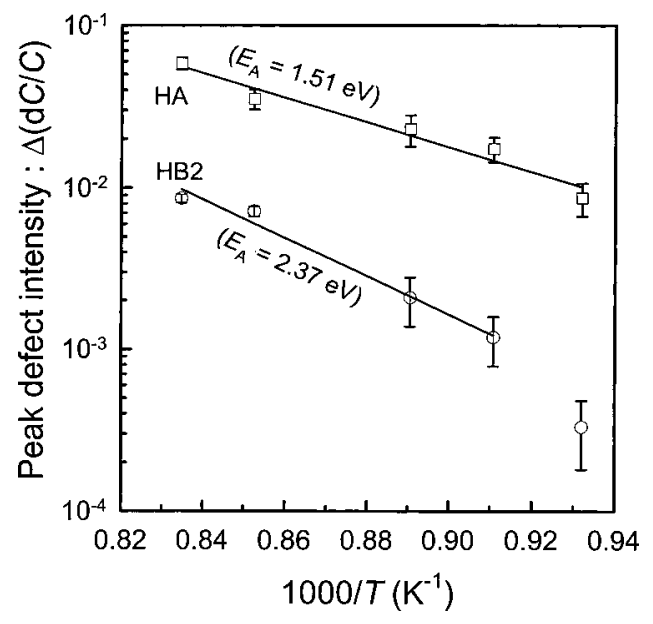

FIG. 3. Arrhenius plots from which the activation energies for the formation of HA and HB2 are determined. The peak defect intensities are obtained from DLTS spectra in Fig. 2.

that $\mathrm{H} 3$ was not the same as HL3 (Fe-related), and proposed it was rather a $\mathrm{As}_{i}$ impurity-related complex. ${ }^{15}$ Despite the fact that the double donor state of EL2 has not previously been observed in epitaxially grown $p$-GaAs layers, the signature of HM1 is quite similar to that of HB2. At this point, we can say that HB2 is probably a defect related to either the $\mathrm{As}_{i}(\mathrm{H} 3)^{15}$ or $\mathrm{As}_{\mathrm{Ga}}$ (HM1), ${ }^{16}$ which is consistent with our present understanding of IFD in GaAs. ${ }^{11-13}$

Figure 3 illustrates the Arrhenius-like dependence of the peak defect intensity of HA (open squares) and HB2 (open circles) on the inverse of annealing temperature. The solid lines are least-square fits to the experimental data points. The linear fit for $\mathrm{HB} 2$ does not include the experimental data point for $800^{\circ} \mathrm{C}$, since the DLTS peak was in fact intermediate between that of HB1 and HB2 (see Fig. 2). Activation energies of $\sim(1.5 \pm 0.3) \mathrm{eV}$ and $\sim(2.4 \pm 0.3) \mathrm{eV}$ are obtained for the creation of HA and HB2. These energies should be treated with caution since they include several components, such as the energy of formation of the constituents of the defect complexes, the energies associated with the diffusion of these constituents and the creation of HA and HB2. These defects are created under nonequilibrium conditions, which limit comparison of our results with energies usually measured under thermal equilibrium conditions.

We now explain the changes in $N_{A}$ shown in Fig. 1. HA can be identified as the $\mathrm{Cu}_{\mathrm{Ga}}$ double acceptor state, ${ }^{14,17}$ which behaves as a $p$-type dopant in GaAs. ${ }^{17-19}$ We have used photoluminescence measurements (not shown) to confirm the identity of $\mathrm{Cu}$ in $p$-GaAs layers. $\mathrm{Cu}$ diffuses rapidly even at low temperatures presumably as an interstitial species $\left(\mathrm{Cu}_{i}\right)$, while substitutional $\mathrm{Cu}$ (i.e. $\mathrm{Cu}_{\mathrm{Ga}}$ ) is relatively immobile. ${ }^{17-19}$ The formation of $\mathrm{Cu}_{\mathrm{Ga}}$ may proceed by either the kick-out process $\left(\mathrm{Cu}_{i}+\mathrm{Ga}_{\mathrm{Ga}} \rightarrow \mathrm{Cu}_{\mathrm{Ga}}+\mathrm{Ga}_{i}\right)$ or the substitutional process $\left(\mathrm{Cu}_{i}+V_{\mathrm{Ga}} \rightarrow \mathrm{Cu}_{\mathrm{Ga}}\right)$. Although the kick-out mechanism is the favored model for the diffusion of $\mathrm{Cu}$, we speculate that the vacancy-assisted process may become dominant under the nonequilibrium injection of $V_{\mathrm{Ga}}$ during IFD. Since $\mathrm{Cu}$ has the tendency to decorate open volume defects in GaAs, ${ }^{19}$ we propose that the injection of excess $V_{\mathrm{G}}$ provides the driving force for the segregation of $\mathrm{Cu}$ towards the surface. $\mathrm{HB} 2$ is related to the creation of an 
excess $\mathrm{As}_{i}$ in the near-surface layer of $p$-GaAs due to the outdiffusion of $\mathrm{Ga}$ atoms into the $\mathrm{SiO}_{2}$ layer. The concentration of hole traps in disordered samples is too low to account for the marked increase in $N_{A}$ depicted in Fig. 1.

We, therefore, arrive at the conclusion that the pronounced increase in the doping concentration in the nearsurface region of disordered $p$-GaAs epilayers is mostly due to the redistribution of $\mathrm{Zn}$. The segregated $\mathrm{Zn}$ in the top $\sim 0.5 \mu \mathrm{m}$ layer could come from the underlying epilayer and/or the $p^{+}$-substrate. Although there is no overwhelming evidence for the exact mechanism that is responsible for $\mathrm{Zn}$ diffusion in GaAs, the kick-out mechanism is the generally accepted model (similar to $\mathrm{Cu}$ ) ${ }^{7-10}$ However, it is very difficult to maintain this model under IFD conditions producing a nonequilibrium concentration of $V_{\mathrm{Ga}}$. Indeed, in their investigation of the evolution of a Be (similar diffusion behavior as $\mathrm{Zn})^{7,8}$ marker in GaAs during IFD using $\mathrm{SiO}_{2}$ and $\mathrm{Si}_{3} \mathrm{~N}_{4}$ capping layers, Haddara et al. ${ }^{20}$ concluded that the diffusion process involved time dependent concentrations of both $V_{\mathrm{Ga}}$ and $\mathrm{Ga}_{i}$. Furthermore, Ky et al. ${ }^{9}$ have shown that $V_{\mathrm{Ga}}$ played an important role in the diffusion of $\mathrm{Zn}$ in GaAs doped as low as $\sim 4 \times 10^{16} \mathrm{Zn} / \mathrm{cm}^{3}$ under equilibrium conditions. The results presented here show that both $\mathrm{Cu}$ and $\mathrm{Zn}$ diffuse towards the near-surface of disordered $p$-GaAs epilayers where an excess of $V_{\mathrm{Ga}}$ is injected. Our discussion on the atomic relocation process is not intended to provide definitive answers to the contentious diffusion mechanism of $\mathrm{Cu}$ and $\mathrm{Zn}$. More importantly, it demonstrates that our understanding of IFD in undoped heterostructures cannot be automatically applied to doped structures, and the ways to suppress $\mathrm{Zn}$ relocation should be addressed before IFD becomes a viable technology for device integration applications.

In summary, we have used $C-V$ and DLTS techniques to characterize IFD $p$-GaAs epitaxial layers. Two hole traps $\mathrm{HA}\left(\mathrm{Cu}\right.$ related) and $\mathrm{HB} 2$ (complex of either $\mathrm{As}_{i}$ or $\mathrm{As}_{\mathrm{Ga}}$ ) are created in the disordered layers with activation energies $\sim(1.5 \pm 0.3) \mathrm{eV}$ and $\sim(2.4 \pm 0.3) \mathrm{eV}$, respectively. We have observed $\mathrm{Zn}$ and $\mathrm{Cu}$ segregation towards the surface of the disordered $p$-GaAs epilayers, where a nonequilibrium injection of $V_{\mathrm{Ga}}$ occurs during $\mathrm{SiO}_{2}$-capped annealing. This strong atomic relocation process results in an increased doping concentration in the near-surface region of samples.

Two of the authors (P.N.K.D. and H.H.T.) acknowledge the financial support of the Australian Research Council. The authors are grateful to Dr. M. Buda and Professor R. M. Cohen for fruitful discussions.

${ }^{1}$ D. G. Deppe, L. J. Guido, N. Holonyak, Jr., K. C. Hsieh, R. D. Burnham, R. L. Thornton, and T. L. Paoli, Appl. Phys. Lett. 49, 510 (1986).

${ }^{2}$ S. Bürkner, M. Maier, E. C. Larkins, W. Rothemund, E. P. O'Reilly, and J. D. Ralston, J. Electron. Mater. 24, 805 (1995).

${ }^{3}$ P. N. K. Deenapanray, L. Fu, H. H. Tan, and C. Jagadish, Electrochem. Solid-State Lett. 3, 196 (2000); L. Fu, P. N. K. Deenapanray, H. H. Tan, C. Jagadish, L. V. Dao, and M. Gal, Appl. Phys. Lett. 76, 837 (2000).

${ }^{4}$ P. N. K. Deenapanray, H. H. Tan, M. I. Cohen, K. Gaff, M. Petravic, and C. Jagadish, J. Electrochem. Soc. 147, 1950 (2000); P. N. K. Deenapanray and C. Jagadish, J. Vac. Sci. Technol. B 19, 1962 (2001).

${ }^{5}$ A. Pépin, C. Vieu, M. Schneider, H. Launois, and Y. Nissim, J. Vac. Sci. Technol. B 15, 142 (1997).

${ }^{6}$ P. N. K. Deenapanray and C. Jagadish, Electrochem. Solid-State Lett. 4, G11 (2001).

${ }^{7}$ R. M. Cohen, Mater. Sci. Eng., R. 20, 167 (1997).

${ }^{8}$ S. Yu, T. Y. Tan, and U. Gösele, J. Appl. Phys. 69, 3547 (1991).

${ }^{9}$ N. H. Ky, L. Pavesi, D. Araújo, J. D. Ganière, and F. K. Reinhart, J. Appl. Phys. 69, 7585 (1991).

${ }^{10}$ D. G. Deppe and N. Holonyak, Jr., J. Appl. Phys. 64, R93 (1989).

${ }^{11}$ P. N. K. Deenapanray, H. H. Tan, C. Jagadish, and F. D. Auret, Appl. Phys. Lett. 77, 696 (2000); J. Appl. Phys. 88, 5255 (2000).

${ }^{12}$ P. N. K. Deenapanray, A. Martin, and C. Jagadish, Appl. Phys. Lett. 79, 2561 (2001).

${ }^{13}$ P. N. K. Deenapanray, Bin Gong, R. N. Lamb, A. Martin, L. Fu, H. H. Tan, and C. Jagadish, Appl. Phys. Lett. 80, 4351 (2002).

${ }^{14}$ A. Mitonneau, G. M. Martin, and A. Mircea, Electron. Lett. 13, 666 (1977).

${ }^{15}$ D. Stievenard, X. Boddaert, and J. C. Bourgoin, Phys. Rev. B 34, 4048 (1986).

${ }^{16}$ J. Lagowski, D. G. Lin, T.-P. Chen, M. Skowronski, and H. C. Gatos, Appl. Phys. Lett. 47, 929 (1985).

${ }^{17}$ R. Leon, P. Werner, K. M. Yu, M. Kaminska, and E. R. Weber, Appl. Phys. A: Mater. Sci. Process. 61, 7 (1995).

${ }^{18}$ R. N. Hall and J. H. Racette, J. Appl. Phys. 35, 379 (1964).

${ }^{19}$ R. Krause-Rehberg, K. Petters, and J. Gebauer, Physica B 273, 714 (1999).

${ }^{20}$ Y. M. Haddara, M. D. Deal, and J. C. Bravman, Appl. Phys. Lett. 68, 1939 (1996). 